\title{
In search of a cause of cryptogenic fibrosing alveolitis (CFA)*: one initiating factor or many?
}

Margaret Turner-Warwick

Emeritus Professor of Medicine, University of London, London, UK

\begin{abstract}
Introductory article
Detection of adenovirus E1A DNA in pulmonary fibrosis using nested polymerase chain reaction
\end{abstract}

K Kuwano, Y Nomoto, $R$ Kunitake, $N$ Hagimoto, $T$ Matsuba, Y Nakanishi, $N$ Hara

The history of patients with idiopathic pulmonary fibrosis (IPF) shows that the disease may be preceded by a viral-like illness. Although viruses have not been demonstrated, it is possible that viruses were not detected in culture because they do not replicate during latency. We investigated the presence of adenovirus in IPF and interstitial pneumonia associated with collagen vascular disease (CVD-IP), using the nested polymerase chain reaction (PCR) and in situ hybridization (ISH) for the E1A region of the adenovirus genome. Studies were performed on lung tissues obtained by transbronchial lung biopsy from 19 patients with IPF, 10 patients with CVD-IP and, for comparison, 20 patients with sarcoidosis. The E1A DNA was present in 3 out of $19(16 \%)$ cases of IPF, in 5 of $10(50 \%)$ cases of CVD-IP, and in 2 of $20(10 \%)$ cases of sarcoidosis. The incidence of E1A DNA in CVD-IP was significantly higher than that in sarcoidosis $(p<0.05)$. In patients with IPF and CVD-IP, E1A DNA was more prevalent in patients treated with corticosteroids (6 out of 9 cases; $67 \%$ ) than in those without it ( 2 out of 20 cases; $10 \%)(p<0.01)$. ISH studies showed that 1 out of 8 cases of IPF and CVD-IP, in which E1A DNA was detected by PCR, was positive for E1A DNA. We conclude that adenovirus E1A is unlikely to be aetiologically involved in the pathogenesis of idiopathic pulmonary fibrosis or interstitial pneumonia associated with collagen vascular disease. However, a latent adenovirus infection may be reactivated or may newly infect the host following corticosteroid administration. (Eur Respir J 1997;10:1445-9)

The technical aspects regarding adenovirus detection in the study by Kuwano et al, ${ }^{1}$ including the negative E1A polymerase chain reaction (PCR) controls and the nested PCR for E1A DNA which was shown to be some 100 times more sensitive than conventional PCR, seem quite satisfactory. Unfortunately, normal control lung quite satisfactory. Unfortunately, norm
tissue was not included in this study.

However, Matsuse et $a l^{2}$ had previously demonstrated E1A DNA in $87 \%$ of paraffin embedded lung samples obtained from patients undergoing resection for lung cancer. They also found E1A DNA in $90 \%$ of tissue samples from smokers with COPD compared with $44 \%$ of smokers without COPD. In view of the frequency of from normal subjects Dif ifficult to understand why the authors postulated that, using the same techniques, they might be able to demonstrate adenovirus sig-

* Interstitial pulmonary fibrosis (IPF) is the preferred term in North America and will be used interchangeably with the term cryptogenic fibrosing alveolitis (CFA) largely used in Europe. nificantly associated with idiopathic pulmonary fibrosis (IPF).

Far from finding an association of E1A DNA with IPF, the authors of the introductory article found the incidence in both treated and untreated cases was significantly less than in previous normal controls or patients with COPD. Since small transbronchial biopsy patients with COPD. Since small transbronchial biopsy specimens were used in the IPF studies, sampling might
account for part of the low yield. Alternatively, the account for part of the low yield. Alternatively, the
authors suggest that, since the E1A region of adenovirus is responsible for sensitising infected cells to cytolysis by tumour necrosis factor (TNF), natural killer (NK) cells and activated macrophages, this might lead to the elimination of virus by the host's immune response. The finding of a somewhat higher prevalence of virus in (but in than in norma controls) where immune responses might be reduced, could support such an explanation. It is conceivable that an active adenovirus infection might be an initiating event but that the consequential inflammatory response eliminates the virus so that it can no longer be detected while other host derived factors, yet to be defined, account for the continuing inflammatory and fibrosing 
response in the lung. If patients could be studied shortly after their virus-like episode, more evidence might be obtained. This, however, is likely to be very difficult because viral-like illnesses are very common but very few patients subsequently develop IPF. The lack of response to the antiviral agent ribavarin in advanced cases of $\mathrm{IPF}^{3}$ suggests that viral agents are not contributing to the progression of late stage disease, irrespective of whether or not they are involved as an initiating agent.

\section{Implication of other viruses}

This topic has recently been reviewed in considerable detail by Egan $e t a l^{4}$ and one or two interesting pointers will be outlined briefly here. There has recently been will be outlined briefly here. There has recently been
considerable interest in Epstein-Barr virus (EBV). Its considerable interest in Epstein-Barr virus (EBV). Its
implication in IPF was first suggested in $1984^{5}$ when Vergnon et al found raised levels of immunoglobulins $\mathrm{G}$ and $\mathrm{A}$ to viral capsid antigen (VCA). A more recent study ${ }^{6}$ explored the possibility of identifying replicating EBV in tissue samples and demonstrated positive staining using immunohistochemical techniques for VCA ing using immunohistochemical techniques for
and the membrane antigen gp $340 / 220$. Positive staining
was found in 14 of 20 patients with IPF but in only two was found in 14 of 20 patients with IPF but in only two
of 21 control samples taken from lung tissue removed at of 21 control samples taken from lung
the time of resection of lung cancers.

The same authors also studied the presence of EBV DNA using nested PCR and obtained positive results in 11 of 20 cases of IPF compared with one of eight normal lungs and one of eight cases of sarcoidosis.?

However, these findings were not confirmed by another However, these findings were not confirmed by another
group ${ }^{8}$ who studied 12 patients with CFA, two with group $^{8}$ who studied 12 patients with CFA, two with
associated systemic sclerosis, three with diffuse alveolar associated systemic sclerosis, three with diffuse alveolar
damage, and one each with desquamative interstitial pneumonitis (DIP), extrinsic allergic alveolitis (EAA), and idiopathic haemosiderosis. EBV DNA was sought using PCR amplification and EBV RNA using in situ hybridisation (ISH) and immunohistochemistry to hybridisation (ISH) and immunohistochemistry to
identify three well defined EBV antigens (VCA, BZ-1, identify three well defined EBV antigens (VCA, BZ-1,
and CS1-4). While EBV antigen staining was positive and CS1-4). While EBV antigen staining was positive
in a patchy distribution in the majority of samples and throughout the different types of lung diseases studied, EBV DNA was identified in small amounts on one occasion in only three samples (two CFA and one foregut cyst) but was not identified on repeat sampling. The resol studies might be helped by exchange of further samples, by further work to standardise and optimise the difficult techniques employed, and by studying larger groups of well defined patients.

Other viruses have also been suggested. Ueda et at found antibodies to hepatitis C (HVC), an RNA virus, in $28 \%$ of patients with IPF but this may have been due to a high number of false positive results and the high background rate of HVC infection. Currently there seems background rate of HVC infection. Currently there seems
to be little evidence of a specific relationship with CFA.
The possibility remains that one or more virus inThe possibility remains that one or more virus infections may initiate inflammation which may lead on to a fibrosing process, either as a result of a $\mathrm{T}$ cell mediated inflammatory response or, alternatively, by transforming smooth muscle cells or fibroblasts resulting in a phenotype which expresses genes involved in the synthesis of matrix proteins or profibrotic mediators. ${ }^{10}$

\section{Occupational "causes" of patients previously} labelled as CFA

While many minerals and dusts can be associated with the general clinical, radiographic, and pathological fea- tures of "pulmonary fibrosis", they often show features which distinguish them from CFA including asbestos, silica, coal, graphite, hard metal, avian proteins and pharmaceutical drugs. ${ }^{11}$ It is therefore necessary to exclude all possible "external causes" before making a diagnosis of CFA. to such exclusions has varied. Some clinicians will exclude cases where there is a potential external causal factor suggested by a substantial occupational history and especially when the lung biopsy or bronchoalveolar lavage sample shows a heavy burden of dust or mineral, even when the putative agent is not currently formally accepted as a cause of pneumoconiosis for compensation purposes. Other clinicians will only exestablished causes of pneumoconiosis, classifying all other cases as "cryptogenic". Either approach is justifiable, depending on the purpose of the studies. However, when the latter approach is taken, and in view of the wide range of agents associated with the development of pulmonary fibrosis, it is not surprising that systematic searches for occupations with exposure to various dusts reveals patients provisionally diagnosed as having "cryptogenic" patients provisionally

In a preliminary case-control study Scott $e t ~ a l^{12}$ re-
fibrosing alveolitis. In a preliminary case-control study Scott et $\mathrm{al}^{12}$ re-
ported evidence of an association between IPF and wood and metal dusts. This work was extended ${ }^{13}$ in a further case control study in which 218 patients diagnosed as having CFA were compared with 569 controls Their life ine and sex by questionnaire and telephone interview. After adjusting for smoking there was a significantly increased relative risk for exposure to a wide range of metals as well as a wide variety of woods. A significant increased relative risk to specific metals was only found for lead, brass and steel; pine was the only specific wood identified. In both groups the exposure history was substantial, extending over years. While the risk increased with duration in years of exposure to metals, oddly the risk decreased with length of exposure to wood dust. ${ }^{14}$ The study concluded that the combined fraction of both wood and metal exposure in this series might account for about $20 \%$ of cases of CFA $(10-13 \%$ of cases exposed to metal dusts and $5-10 \%$ of those exposed to wood dusts). These results are supported by a case control study on necropsy material from 1137 cases in whom IPF was significantly related to those exposed to metal and wood as well as laundry workers, barbers, beauticians and painters. ${ }^{15}$ In a further case-control study by the same authors in which 86 living patients with IPF were compared with hospital controls there was a significant relationship with those with a history of working with metal and in mines. Another recent study in which 248 patients with IPF were compared with age, sex and geographically matched controls found a significant association in those involved with farming, hairdressing, exposure to metal dust, raising birds, stone cutting and exposure to animal and vegetable dusts. ${ }^{16}$

As suggested above, because of the substantial exposures likely to have been recorded in the routine clinical history, some clinicians might not have included clinical history, some clinicians might not have included some of these cases under the heading "cryptogenic"
but rather flagged them as possibly associated with dust but rather flagged them as possibly associated with dust
exposure, especially if the biopsy or lavage specimen was heavily laden with dusts. Furthermore, where macrophages are heavily laden with dust particles or where interstitial deposits are found, many pathologists would proceed to birefringent studies, electron microscopy, or even studies using analytical microprobes. For 
example, a study by Johnson $e t a l^{17}$ identified particles which were related to known exposures, superimposed on a background of other particles relating to smoking (kaolinite and mica) and to the general environment (silicon, titanium and iron). The study was particularly helpful when light microscopy indicated retention of dust in patients with no known history of exposure. In this context it would be interesting to know whether, in the multicentre case control study discussed above, ${ }^{13}$ the substantial exposure identified on the systematic questionnaire had also been recorded in the initial clinical case history. It would also be interesting to know how often biopsy or lavage was performed, whether these samples showed a heavy dust burden, and whether this had triggered the pathologists to ask for further details. It would be particularly interesting if tissue or lavage material was available which failed to find inorganic material or other particles in these lungs on light microscopy. A case has recently been reported of a carpenter with clinical CFA who had been exposed to fibreglass in whom fibreglass was only identified by analytical electron microscopic examination and was not identified on routine light microscopy or by birefringence. ${ }^{18}$

One of the problems with the current trend of making the clinical diagnosis of CFA supported by CT scan but without supporting pathological material or bronchoalveolar lavage (BAL) samples is that the suspicion of such initiating agents may be overlooked.

However, notwithstanding these specific points, there seems to be increasing evidence that a wide range of external dusts including some metals and wood not yet officially accepted as causing pneumoconiosis may contribute in some way - either as an initiating agent or as a contributing factor - in a few cases of pulmonary fibrosis previously categorised as being of "unascertained" cause. This fraction will be smaller in series of patients where some would have been exclude the basis of their possible dust exposure. It is also likely to be smaller in populations which in general are less exposed to external dusts of these types.

The identification of exposure to a very wide variety of metals, woods and other dusty materials with a higher risk being associated with only very few specific materials, and the fact that these exposures account for only a small number of cases of CFA, perhaps favours the suggestion that these dusts may act in a non-specific way suggestion that these dusts may act in a non-specific way as contributing factors in genetically or non-genetically
predisposed individuals, rather than acting as specific

predisposed ind
initiating agents.

Many reports have observed a high frequency of smoking amongst patients with CFA; $74 \%$ of patients in an early large survey were smokers or ex-smokers ${ }^{1}$ eris factor has been confirmed more recently. ${ }^{20}$ The response to corticosteroids also seems to be less good in smokers, ${ }^{21}$ but whether or not this is another example of a non-specific dust risk factor or whether the components of cigarette smoke, perhaps by recruiting neutrophils to the lungs, ${ }^{22}$ contribute more specifically by promoting inflammation initiated by some other agent remains unproven.

Possible implication of genetic factors modulating immunological responses, inflammation, and fibrogenesis

Several facts suggest the implication of genetic factors in the development of fibrosing lung disease. For example, familial CFA is well recognised but many of these cases present in infancy and in this respect are distinct from adult IPF. ${ }^{102324}$ The genetic basis for this group may therefore be different. Of particular interest is the observation in three families with familial IPF where nonaffected members also had increases in the levels of various inflammatory components in BAL fluid samples including neutrophils, activated macrophages, and fibroblast growth factors. ${ }^{25}$ The genetic factor or factors determining these changes has not yet been identified. In fibrosing lung disease due to a variety of known occupational exposures such as beryllium and asbestos not all individuals with apparently similar exposure develop the disease, suggesting a genetic or non-genetic predisposition. In searching for "causes" it should not (hen there is a clearly defined cause supported by experimental challenge studies showing a "linear" dose-response curve measuring injury, this demonstrates two things rather than one. On the one hand it indicates the strong influence of the agent on the development of the disease under study but, in addition, it also demonstrates that at points along the dose-response curve subjects receiving the he disease, depending on variations in genetic as well as non-genetic factors. For example, there is now considerable evidence that genetic host factors play a dominant role in susceptibility at low doses of exposure but that injury due to the external agent predominates at high doses. ${ }^{26}$ Even in experimental studies using inbred strains of animals the intrinsic susceptibility of the species and inherent non-genetic influences will both influence the doseresponse relationship to external agents. ${ }^{27}$

The major histocompatibility complex (MHC) located on chromosome 6, encoding HLA molecules on the surface of presenting cells, is functionally associated with $\mathrm{T}$ cell receptor $(\mathrm{TcR})$ recognising foreign proteins. This is the driving force of the immune response and results in $\mathrm{T}$ cell activation, cytokine production, and antibody formation. ${ }^{2324}$ Although reports on the asrecilition association of $\mathrm{MHC}$ and CFA remain conflicting, ${ }^{2829}$ results from studies on fibrosing alveolitis in systemic
sclerosis (FASSc) are much clearer. For example, Briggs sclerosis (FASSc) are much clearer. For example, Briggs
et $a l^{30}$ have shown HLA-DR3/DR52a alleles and/or the anti-Scl-70 autoantibody to be risk factors for the development of FASSc. Another study identified the TGF $\beta$ gene - a growth factor with many functions including fibrogenesis polymorphism (A25P) - to be including fibrogenesis polymorphism (A25P) - to

The genes within the MHC region are highly polyThe genes within the MHC region are highly poly-
morphic and are associated with autoimmune disease morphic and are associated with autoimmune disease.
The clinical picture of CFA is well recognised in association with many cases of systemic sclerosis and some, albeit less commonly, with rheumatoid arthritis and systemic lupus erythematosus. Furthermore, there is an increased in antibodies (rheumatoid factor and antinuclear antibody) in patients with "lone" CFA..$^{32}$ A very recent study using new molecular techniques has demonstrated specific autoantibodies to type II pneumocytes in CFA.

Evidence for the implication of $\alpha_{1}$-antitrypsin, the potent inhibitor of neutrophil protease in the development of fibrosis, in rheumatoid arthritis is conflicting. Geddes et $a^{34}$ demonstrated that possession of the non-MM for $\alpha_{1-}$ antitrypsin, especially $M Z$, was associated with the development of pulmonary fibrosis in patients with rheumatoid arthritis. This finding was not, however, confirmed by Steers et al. ${ }^{35}$ Larger numbers need to be studied to resolve this question.

The field of genetics in fibrosing lung disease is now rapidly developing and is of great potential importance. 
It should help our understanding of pathogenesis, including immunological and host inflammatory responses in fibrosing lung disease, the susceptibility to fibrosis, the rate of progression of fibrosis, and the prognosis. There have been several useful reviews covering the current knowledge in this field. ${ }^{102436}$

\section{Important general considerations in attempting} to find new "causes"

In order to evaluate the recent attempts to identify causal agents in cryptogenic fibrosing alveolitis, it may be useful to restate some of the inherent problems when working in this difficult field. In doing so, some ideas on possible new approaches will be made.

The aetiological factors in any disorder defined in clinicopathological terms include the initiating agent(s) - for brevity referred to in this article as "causes" contributing factor(s), and the consequential host cellular and humoral responses. The interaction between these three sets of factors is complex. When initiating agents are difficult to find it is understandable but often misleading to attribute a "causal" role to a factor which is more properly a contributing factor or part of the is more prope
host response.

It is not uncommon for a disorder defined in clinicopathological terms to have more than one initiating agent. It is also well recognised that distinctive conditions, often with a known cause and well characterised host responses, progress to a final common path of pathological changes which determine a common set of clinical features at this late stage. When in clinical practice patients only present at a late stage, it is understandable to regard this as a single disease entity albeit with one or more "causes".

When, as is common in medicine, there is no identifiable cause, a disorder has to be defined in descriptive terms. Where the characterising features are present in all the patients, which unfortunately is rarely the case, all the patients, which unfortunately is rarely the case,
research into causes and pathogenesis of such a homoresearch into causes and pathogenesis of such a homo-
geneous group can be built on a firm base. However, geneous group can be built on a firm base. However, when the definition is based on a cluster of clinico-
pathological features which distinguishes the group of patients from others, but in which individuals do not necessarily have all the features, research into causation of these heterogeneous groups of patients becomes much more difficult. When, for example, there is a range of histological patterns in a clinically defined group, do these represent different stages of the same condition, do they indicate subgroups with different initiating factors, or does the variation in histology reflect the influence of contributing factors or host responses (to a single or several causes)?

In order to dissect out definitively the role of all these variables in search of causation, large numbers of cases are necessary. However, when the disorder is relatively rare answers are very difficult to obtain unless some very strong and clearcut evidence emerges. Further, when attempts are made to increase numbers by undertaking multicentre studies on these heterogeneously defined groups, there is no certainty that the variables are evenly distributed between the different centres. For example, environmental and occupational factors may vary considerably between rural and urban centres or vary considerably between rural and urban centres or
the referrals to some centres may have a bias towards the referrals to some centres may have a bias towards
late stage disease. Prospective studies with tightly agreed late stage disease. Prospective studies with tightly agreed
inclusion and exclusion criteria and with equally tight clinical and laboratory protocols should help, but these have encountered many practical difficulties even when attempted.
All of these problems are very relevant to the recent reports attempting to define some of the possible initiating factors in CFA.

\section{Clarification of terminology in relation to known} and unknown causal factors

The general term "interstitial pulmonary fibrosis" based on pathological features characterises a lung tissue response to a range of known external agents as well as those with an unidentified cause, resulting in injury to any part of the lung which heals with a tendency towards fibrosis rather than resolution. If the definition is narrowed to specify the charact rowed to specify the chacteristic of lower zone predominance, the range of initiating agents is reduced but still includes asbestos, coal dust (typically those in which " $p$ " appearances are evident on the radiograph (ILO classification)), graphite, ${ }^{37}$ some unusual cases of silica, ${ }^{38}$ and hard metal. ${ }^{39}$ This list is not yet complete and new agents are continually being suggested. ${ }^{13} 18$

However, and only for the time being, there are groups of patients with many clinical features in common which show a similar fibrosing histological pattern in which no cause has yet been identified; these were initially labelled as IPF. Although meaning "unknown", implying that with further knowledge more causes would be identified, the term has sometimes been used to imply that IPF represents a "disease entity" where there is no initiating agent. In order to stress the importance of recognising our curren of recow " ledge, the term "cryptogenic" ("hidden") was introduced to stress the likelihood of identifying more causes in the future, hopefully and eventually leading to the elimination of the term. The qualifying adjective "fibrosing" was introduced to describe more accurately the histological features which almost always included an inflammatory response of varying degree as well as established fibrosis. ${ }^{40}$

Further difficulties have arisen as more histological variants have been identified in patients presenting with the same - albeit crudely described - clinical, physiological, and radiographic features. Although it is not yet complete, there seems to be increasing evidence that histological variants such as desquamative interstitial pneumonitis (DIP) ${ }^{41}$ giant cell pneumonia (GIP) which is now recognised as frequently being associated with hard metal exposure, ${ }^{41}$ and even lymphocytic interstitial pneumonia (LIP) which is often associated with systemic lymphoproliferative disease ${ }^{42}$ and some viral infections, ${ }^{43}$ are distinctive conditions although they may progress in at least some patients to an advanced fibrosing stage, histologically similar to usual interstitial pneumonia (UIP) as described by Liebow. ${ }^{44}$

Recently a new approach relating to pathogenesis has been suggested. ${ }^{45}$ Cases in which the histological appearances, which may be cellular, mixed or fibrotic, appear to be distributed evenly and uniformly across the whole of the biopsy specimen led the authors to suggest that the appearance reflected a single initial insult in time. They proposed the term "non-specific interstitial pneumonia" (NSIP) to distinguish these cases from UIP characterised by a patchy mixing of cellular, fibrotic and destructive features which, they suggested, reflected repeated injury extending over periods of time. The practical value of this approach is that cases showing features of NSIP seem to have a better survival and respond more readily to treatment. ${ }^{4546}$ Earlier studies have shown that CFA with a more "cellular" histological pattern has a better prognosis and responds better to treatment. ${ }^{47}{ }^{48} \mathrm{It}$ is now 
important to see whether the criteria previously used to define a "cellular" pattern correspond to those of nonfibrotic NSIP. It seems that the fundamental factor determining a better prognosis and response to treatment may depend more on whether the alveolar arment may depend mite on 作 classified in so many different ways. On the other hand, where the alveolar units are destroyed ("honeycombing”) then prognosis is less good. Adequate sized biopsy specimens are essential to establish this and CT scans are especially helpful.

In clinical practice the value of obtaining histological material in presumed CFA is twofold. Firstly, it can exclude a range of other pathologies which can mimic fibrosing alveolitis and secondly, as discussed above, it can indicate the likely prognosis and responsiveness to treatment. If the latter fundamentally depends on whether the lung architecture is intact or destroyed, then high resolution computerised tomography (HRCT) should provide a non-invasive way of establishing this. Several studies have now demonstrated (49-51 its value in groups of patients ${ }^{49-51}$ the very serious prognosis in most cases of CFA and the difficulties and risks of treatment, a good case remains for obtaining both histological material and radiographic evidence to exclude other conditions until the reliability of CT scanning in all individual patients has been proved beyond doubt.

Some special features of CFA yet to be explained and requiring more research

When attempting to identify new causes of CFA it is worth emphasising some of the special features which, although not present in every case, distinguish this group of patients from other types of pulmonary fibrosis. These features require explanation and are important because they may stimulate ideas for new lines of research on they may stimulate ideas for new lins
the role of various aetiological factors.

DISTRIBUTION OF DISEASE

The disease is predominantly located in the peripheral parts of the lower zones. The distribution is not segmental and does not seem to follow bronchial, vascular, or lymphatic pathways. This distribution is also found in some cases related to inhaled particles, especially asbestos. In cases with minimal involvement - which may or may not be the same as early disease - HRCT scanning has shown that the changes are first seen under the parietal pleural surface of the lung and then progress to involve more central areas. This requires explanation.

FINGER CLUBBING

Many different reports agree that, in contrast to many other types of pulmonary fibrosis, $40-50 \%$ of cases have finger clubbing. ${ }^{19}$ The explanation for finger clubbing is unknown but its association with proliferation of the bronchial vasculature described more than 30 years ago ${ }^{52}$ and, as far as I am aware, not yet confirmed by others, indicates that new studies on this subgroup using new indicates that new studies on this subgroup using new
knowledge and techniques on angiogenesis might give knowledge and techniques on angiogenesis might give
new insight into the progression of fibrosis and may even new insight into the progression of fibrosis and may even open new lines of treatment. A recent study using extracts from lung biopsy specimens suggests that there is an imbalance between higher than normal levels of interleukin 8 (IL-8) and reduced levels of interferon $\gamma$ (IFN $\gamma$ )-inducible protein (IP-10) in CFA favouring angiogenesis. ${ }^{53}$
“EARLY” DISEASE

In an attempt to study the earliest changes, electron microscopy was used to study the ultrastructure of the most normal parts of the lung in biopsy specimens from subjects with CFA. ${ }^{54}$ This indicated damage to both pulmonary capillary endothelium and pulmonary epipulmonary capillary endothelium and pulmonary epithelial cells in the absence of inflammatory cell infiltration. Furthermore, the studies suggested that this early destruction of epithelial cells was followed by an intra-alveolar exudate which became organised and incorporated into the alveolar walls with repair by type II cells growing over it. These observations throw a rather different light on early pathogenesis from the more conventional emphasis on mphis on the inflammatory cell response which may be a later event. If, as seems probable, epithelial and endothelial damage represents the earliest changes in CFA, then it is important to focus on the pulmonary endothelium and epithelium.

LUNG PERMEABILITY

Following this line of argument, serial non-invasive studies of lung permeability (reflecting epithelial damage in particular) have been undertaken and have shown that restoration of normal permeability with treatment is associated with a clinical response to treatment and continued increased permeability is associated with deterioration. $^{55}$

RAPID PROGRESSION OF DISEASE

Most reports agree that the median duration of disease from presentation to death is rapid over about five years. This dramatic progression of the inflammatory and fibrotic response with increasing architectural destruction of alveolar units and a poor response to treatment in most cases is one of the most characteristic The potential importance of contributing factors such as inhaled environmental dusts and cigarette smoke, and genetic factors especially relating to control of host responses, are now under intensive investigation.

CFA WITH AND WITHOUT "COLLAGEN VASCULAR DISEASE" The debate continues as to whether the fibrosing alveolitis found in association with a variety of so-called collagen vascular diseases - especially systemic sclerosis, rheumatoid arthritis, and systemic lupus erythematosus (SLE) - should be regarded as an entity which is distinct from lone fibrosing alveolitis. The arguments are somewhat spurious. On the one hand there is considerable evidence that a large number of clinical, physiological, radiographic, and pathological features are virtually identical and are shared by both conditions but, on the other hand, there are a number of features that are quite distinctive. For example, a formal study comparing the histological features of FASSc with lone fibrosing alveolitis on light microscopical study found them indistinguishable. ${ }^{56}$ However, systematic studies have now confirmed that the rate of progression in FASSc is much slower. ${ }^{7758}$

Non-organ specific autoantibodies including antinuclear antibody and rheumatoid factor are found in both fibrosing alveolitis associated with collagen vascular diseases (FA-CV) and lone fibrosing alveolitis, albeit with quantitative differences. ${ }^{59}$ However, antibodies to a wide range of other cell component antigens are clearly distinctive both in FA-SLE and FASSc. As noted above, recent studies have shown distinctive genetic aberra- 


\section{LEARNING POINTS}

* When searching for new causes of a condition currently defined in descriptive terms it is important to distinguish between initiating factors, contributing factors, and host responses which may be genetic or non-genetic.

* Several different initiating agents may induce patterns of host response so that there is progression to a final common path of tissue change giving rise to a common pattern of histology which, in turn, determines the common clinical, radiographic, and physiological features. It cannot therefore be assumed that the final common path represents a "disease entity" with a single cause.

* Within a group of patients defined descriptively, not every patient exhibits all the features. This heterogeneity must be borne in mind when seeking new causes and studying genetic factors or pathogenetic mechanisms. Subgroup analysis therefore becomes important. Because CFA is relatively uncommon, multicentre studies will be essential to study sufficient numbers of cases.

* Where research involves complex techniques, exchange of material between centres at an early stage to cross validate results can often advance knowledge more rapidly and avoid non-productive lines of investigation based on spurious results arising from purely technical differences.

tions. ${ }^{30}$ Thus a more practical and constructive approach is to use FA-CV and lone fibrosing alveolitis as useful comparative models in studies to elucidate the various common and distinctive features requiring explanation.

CFA AND LUNG CANCER

Some studies have shown an association with lung cancers not entirely accounted for by smoking. ${ }^{60}$ An excess of lung cancers is also found in asbestosis and in CFA associated with systemic sclerosis. ${ }^{61}$ Thus, the pathogenesis of CFA needs to be considered in relation to oncogenesis with regard to the role of viruses, various growth factors, and genetic predisposition.

\section{Conclusion}

It should be emphasised that many of these characteristic features are likely to depend more on host responses to lung injury than to relate directly to initiating agents or contributory factors. However, they may hold important clues to factors which distinguish this serious and progressive condition from other types of widespread pulmonary fibrosis.

1 Kuwano K, Nomoto R, Hagimoto N, et al. Detection of adenovirus Eur Respir F 1997; 10:1445-9. Matsuse T, Hayashi S, Kuwano K, et al. Latent adenoviral infection in
the pathogenesis of chronic airways obstruction. Am Rev Respir Dis

Agusti C, Xaubet A, Ballester E, et al. Aerosolised ribavarin in patients

with advanced cryptogenic fibrosing alveolitis: a pilot study. Thorax

4 Egan JJ, Woodcock AA, Stewart JP. Viruses and idiopathic pulmonary

fibrosis. Eur Respir F 1997;10:1433-7.

and 6 Egan JJ, Stewart JP, Hasleton PS, et al. Epstein-Barr virus replication within pulmonary epithelial cells in cryptogenic fibrosing alveolitis.
Thorax 1995;50:1234-9. Egan JJ, Stewart JP, Hasleton PS, et al. EBV PRC (Raji I) in idiopathic pulmonary

Wangoo A, Nicholson AG, Diss TC, et al. Cryptogenic pulmonary
fibrosis does not appear associated with Epstein-Barr virus infection. 9 Ueda $\mathrm{T}$, Ohta $\mathrm{K}$, Suzuki $\mathrm{N}$, et al. Idiopathic pulmonary fibrosis and
high prevalence of serum antibodies to hepatitis $\mathrm{C}$ virus. $A m$ Rev Respir Dis 1992;146:266-8. fibrosis: is there a fibrosis gene? Int 7 Biochem Cell Biol 1997;29: Parkes WR. Fundamentals of pathogenesis and pathology. In: $O c$ cupational lung disorders. London: Butterworths, 1982;77:190. A case control study of environmental exposure to dust. $B M \mathcal{F} 1990$

301:1015-7.
13 Hubbard R, Lewis S, Richards K, et al. Occupational exposure to metal or wood dust and aetiology of cryptogenic fibrosing alveolitis. Lancel

the aetiology of CFA. MD thesis, Nottingum University, 1905 . ai K, Mori T, Yamada N, et al. Idiopathic pulmonary fibrosis: Crit Care Med 1994;150:670-5

6 Baumgartner KB, Samet JM, Coultas DB, et al. Occupational and Evidemient 1997.145 factors for idipathic pulmonary fibrosis. $\mathrm{Am}$ Johnson NF, Haslam PL, Dewar A, et al. Identification of inorganic dust paticles in bronchoalveolar lavage macrophage by energy dispersive Xray microanalysis. Ach $\mathrm{M}$ Takena $\mathrm{H}$,

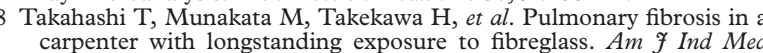
carpenter with $1996 ; 30: 596-600$

19 Turner-Warwick M, Burrows B, Johnson A. Cryptogenic fibrosing alveolitis: clinical

20 Baumgartner KB, Samet JM, Stidley CA, et al. Cigarette smoking: a risk factor for idiopathic pulmonary fibrosis. Am $\mathcal{Y}$ Respir Crit Car

$21 \mathrm{De}$ Cremoux H, Bernaudin JF, Laurent $\mathrm{P}$, et al. Interactions between cigarette smoking and the natural history of idiopathic pulmonary
fibrosis. Chest 1990;98:71-6. fibrosis. Chest 1990;98:71-6.
Schwartz DA, Helmers RA, Dayton CS, et al. Determinants of bronchoalveolar lavage cellularity in idiopathic pulmonary fibrosis. $\mathcal{F} A p p l$
Physiol $1991 ; 71: 1688-93$ 23 Lympany PA, du Bois RM. Diffuse lung disease: product of genetic 24 Lympany PA, du Bois RM. Interstitial lung disease: basic mechanism and genetic predisposition. Monaldi Arch Chest Dis 1997;52:33-6.
25 Bitterman PB, Rennard S. Familial idiopathic pulmonary fibrosis. N 7 Med 1986;314:1343-7.

6 Lympany PA, Haris JM, Dowdeswell $\mathrm{R}$ et al. Interaction of HLA phenotype and smoking with exposure in sensitisation to complex platinum salts. Am $\mathcal{F}$ Respir Crit Care Med 1997;32:A135. (airch' Laboratory Animals) 1995;23:51-60.

28 arpela E, Aillkainen A, Varpela $\mathrm{M}$, $e t$ al. High prevalences of HLALibby DM, Gibofsky A, Fotino $M$, et al. Immunogenetic and clinica findings in idiopathic pulmonary fibrosis. Association with the B-cel

30 Briggs DC, Vaughan RW, Welsh KI, et al. Immunogenetic predictions 1 Lymany PA, Avila JJ, Black CM, et al. TGF $\beta_{1}$ promoter region gene polymorphisms in fibrosing lung disease in aUK pameasod population. Eur Respir $\mathcal{F} 1998$ (in press).
Turner-Warwick M, Doniach

pulmonary fibrosis. $B M 7$ 1965;1:886-91.

Wallace WA, Schofield JA, Lamb D, et al. Localisation of an autoantigen 
In search of a cause of CFA 34 Geddes DM, Webley M, Brewerton DA, et al. $\alpha_{1}$-Antitrypsin phenotypes
in fibrosing alveolitis and rheumatoid arthritis. Lancet 1977;ii: $1049-$

35 Steers G, McMahan MJ, Greenan DM, et al. Lack of association of the articur 36 Coker RK, Laurent G. Pathogenesis of pulmonary fibro

for pharmacological intervention. In: Walters EH, du Bois RM, eds. Immunology and management of interstitial lung diseases. London: Chap37 Gaensler EA, Cadigan IP, Sas

al. Graphite pneumoconiosis 38 Honma J, Chiyotami K. Diffuse interstitial fibrosis in non asbestos pneumoconiosis: a pathological study. Respiration 1993;60:120-6.
Coates EO, Watson JHL. Diffuse interstitial lung disease in to carbide workers. Ann Intern Med 1971;75:709-16.
Scadding JG. Fibrosing alveolitis (letter). BMF 1964;2:686.

1 Liebow AA, Steer A, Billingsley JG. Desquamative interstitial pneuLeibow AA, Carrington CB. Diffuse pulmonary lymphoreticular infiltrates associated with dysproteinaemia. Med

43 Barbera JA, Hayashi S, Hegele RG, et al. Detection of Epstein Barr Liebow AA. Definition and classific

human pathology. Prog Respir Dis 1975;8:1-33. histological features and clinical significance. Am $\mathcal{F}$ Sumg Pathol 1994; 18:136-47. Rvu JH, Edwin MK, et al. Prognostic significance of histopathological subsets of idiopathic pulmonary fibrosis. Am ₹ Respir 7 Johnson MA, Kwan S, Snell NJC, et al. Randomised control trial with low dose prednisolone in cryptogenic fibrosing alveolitis. Thorax 48 Turner Warwick M, Burrows Johnson A. Cryptogenic fibrosing alveolitis:
response to corticosteroid treatment and its effect on survival. Thorax response to corticosteroid treatment and its effect on survival. Thorax
1980;35:593-9. 49 Wells AU, Rubens MB, du Bois RM, et al. Serial CT in fibrosing alveolitis: prognostic significance of the initial pattern. Am $f$ Roentgenol
1993;161:1159-65.
att-Jardin M, Giraud F, Remy J, et al. Importance of ground glass
attenuation in chronic diffuse infiltrative lung disease: CT correlation. Radiology 1993;189:693-8.
Hansell DM, Wells AU. CT evaluation of fibrosing alveolitis: applications and insights. F Thorac Imaging $1996 ;$;11:231 249 .
52 Turner Warwick $M$. Systemic artery patterns in the lung and clubbing of the fingers. Thorax 1963;18:238-50. The CXC chemolines IL-8 and IP-10 regulate angiogenic activity in idiopathic pulmonary fibrosis. F Immunol 1997;159:1437-43.
Corrin B, Dewar A. Pathogenesis of idiopathic interstitial pulmonary 54 Corrin B, Dewar A. Pathogenesis of idiopathic interstital pulmonary
fibrosis. Ultrstructural Pathol 1996;20:369-71.
55 Wells AU, Hansell DM, Harrison NK, et al. Clearance of inhaled Wells AU, Hansell DM, Harrison NK, et al. Clearance of inhaled
$99 \mathrm{mTC}$ T.DTPA predicts the clinical course of fibrosing alveolitis. Eur 6 Harrison NK, Myers AR, Corrin B, et al. Structural features of interstitial
lung disease in systemic sclerosis. Am Rev Respir Dis 1991;144:706-13. 57 lung disease in systemic sclerosis. Am Rev Respir Dis 1991;144:706-13. with systemic sclerosis has a better prognosis than lone cryptogenic
fibrosing alveolitis. Am $\mathcal{Y}$ Respir Crit Care Med 1994;149:1583-90. 5 Papiris PA, Ulachoyiannopoulos PG, Maniata MA, pulmonary fibrosis and pulmonary fibrosis in diffiuse systemic sclerosis
two fibroses with different prognoses. Respiration 1997;64:81-5. 9 Chapman JR, Charles PJ, Venables PJ, et al. Antibodies to nuclea ribonucleoprotein in cryptogenic fibrosing alveolitis; a subset with
mixed connective tissue diseases. Am Rev Respir Dis 1984;130:439-43. 60 Turner Warwick M, Lebowitz M, Burrows B, et al. Cryptogenic fibrosing 61 Richards RL, Milne JA. Cancer of the lung in progressive systemic
sclerosis. Thorax 1958;13:238-45. 